\title{
Acute Exacerbation of Idiopathic Interstitial Pneumonia Triggered by Cardiac Ablation: The First Case Report
}

\author{
Kaho Akimoto, Kuniaki Hirai, Tetsuya Homma, Shintaro Suzuki, \\ Akihiko Tanaka and Hironori Sagara
}

\begin{abstract}
:
Acute exacerbation of idiopathic interstitial pneumonia (AE-IIP) is associated with invasive procedures and respiratory infections. However, there have been no reports of AE-IIP triggered by catheter ablation. We herein report a case of AE-IIP after catheter ablation for atrial fibrillation in an 82-year-old man who was diagnosed with IIP. Cardiac ablation has become an increasingly common procedure for managing patients with arrhythmias. Considering that catheter ablation causes AE-IIP, a detailed clinical interview, physical examination, and chest radiography are necessary before catheter ablation. We should additionally consider AEIIP as a differential diagnosis of respiratory failure after catheter ablation.
\end{abstract}

Key words: idiopathic pulmonary fibrosis, acute exacerbation, catheter ablation

(Intern Med 60: 3589-3592, 2021)

(DOI: 10.2169/internalmedicine.7016-21)

\section{Introduction}

The prognosis of fibrotic idiopathic interstitial pneumonia (IIP) is extremely poor (1). Acute exacerbation (AE) of IIP may be triggered by non-respiratory diseases, such as gastrointestinal surgery and trauma, as well as in those that directly invade the lungs, such as respiratory infections, medications, a surgical lung biopsy, and bronchoscopy $(2,3)$. Cardiac ablation is commonly performed to manage arrhythmias (4); however, no reports have mentioned the relationship between AE-IIP and catheter ablation.

We herein report a case of AE-IIP after catheter ablation.

\section{Case Report}

An 82-year-old man with persistent atrial fibrillation (AF) that developed a year earlier and was refractory to pharmacologic therapy and cardioversion, presented with palpitations. He was undergoing treatment with apixaban, pilsicainide, bisoprolol fumarate for $\mathrm{AF}$, and clopidogrel sulfate and nicorandil for angina.

He underwent cardiac ablation, which involved cryobal- loon ablation for pulmonary vein (PV) isolation. Cryoballoon ablation was performed for each PV for 1-3 minutes (Table 1). All cryoballoon return gas temperatures were recorded at their nadir and ranged from -36 to $-61^{\circ} \mathrm{C}$. The total treatment time for catheter ablation was 165 minutes, and the volume of intravenous fluids received during ablation was $500 \mathrm{~mL}$ of saline. The in/out balance was $-100 \mathrm{~mL}$ during the procedure, and the hemodynamic status was stabilized. Dexmedetomidine and fentanyl were used for sedation and analgesia during ablation, respectively. No difficulty was encountered in mapping and isolating the left superior vein, left inferior pulmonary vein, right superior pulmonary vein, or right inferior pulmonary vein. After ablation, an electrocardiogram showed a normal sinus rhythm. He continued to take medicines other than pilsicainide after cardiac ablation.

He had been diagnosed with fibrotic IIP two years earlier based on the presence of reticulation in the bilateral and lower lung lobes on high-resolution computed tomography (CT) and no findings indicative of secondary interstitial pneumonia complicated with collagen diseases, drugs, and radiation reaction (Figure a). He received no antifibrotic therapy, given his age. His peripheral capillary oxygen saturation was $95-98 \%$ on room air without dyspnea on exer-

Showa University School of Medicine, Department of Medicine, Division of Respiratory Medicine and Allergology, Japan Received: January 4, 2021; Accepted: April 13, 2021; Advance Publication by J-STAGE: June 5, 2021

Correspondence to Dr. Kuniaki Hirai, hiraik@med.showa-u.ac.jp 
Table 1. Recorded Ablation Amounts, Times, and Temperatures.

\begin{tabular}{ccc}
\hline PV & Ablation duration (seconds) & Lowest cryoballoon temperature $\left({ }^{\circ} \mathrm{C}\right)$ \\
\hline LSPV & 180 & -61 \\
LSPV & 180 & -36 \\
LIPV & 90 & -47 \\
RSPV & 180 & -56 \\
RIPV & 180 & -52 \\
\hline
\end{tabular}

PV: pulmonary vein, LSPV: left superior pulmonary vein, LIPV: left inferior pulmonary vein, RSPV: right superior pulmonary vein, RIPV: right inferior pulmonary vein

(a)

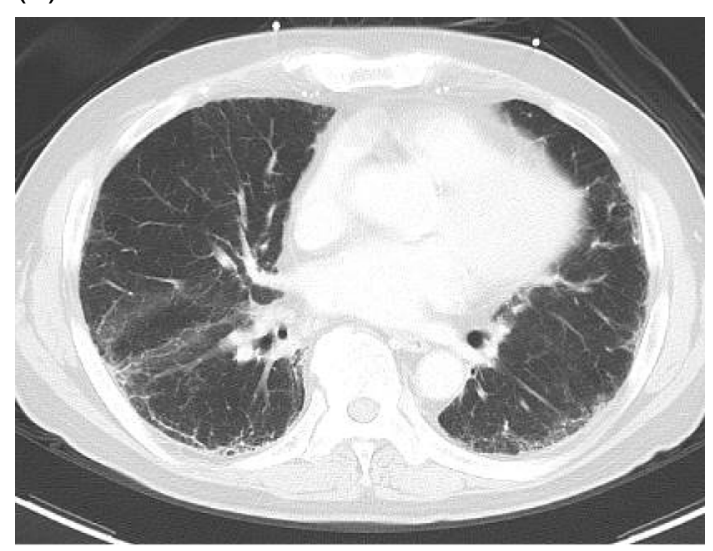

(b)

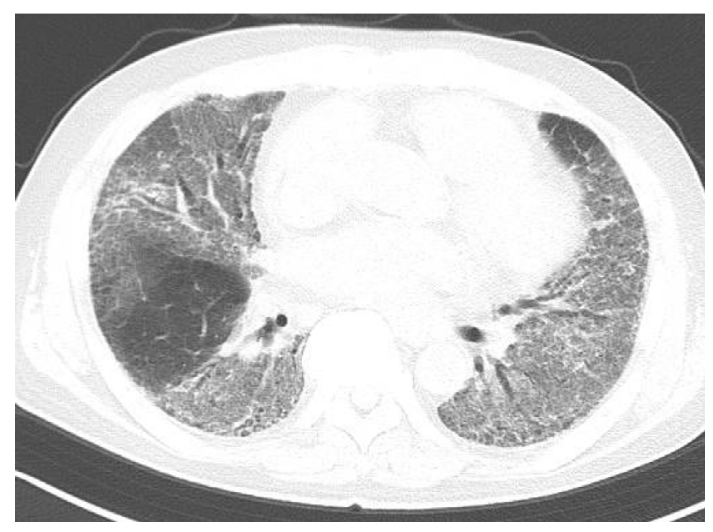

Figure. Chest computed tomography (CT) images. (a) Chest CT before cardiac ablation showed reticulation in the bilateral and lower lung lobes. (b) Chest CT at the time of admission showed new, superimposed ground-glass opacities and consolidation in the bilateral lobes.

tion. The patient's IIP was not considered important until the acute exacerbation, and no close examinations were performed for IIP, such as an arterial blood gas analysis or 6minute walk test. Therefore, the severity of IIP, according to the Japanese Respiratory Society guidelines, was unknown at the time of the ablation.

Five days after ablation, he developed dyspnea. A physical examination revealed fine crackles in the bilateral lower lung fields and finger clubbing. His body temperature was $36.5^{\circ} \mathrm{C}$, blood pressure was $118 / 74 \mathrm{mmHg}$, and his pulse rate was 106 beats per minute. He developed acute respiratory failure (peripheral capillary oxygen saturation of $52 \%$ on room air) and received oxygen therapy. Chest CT revealed new, bilateral, superimposed ground-glass opacities and consolidation (Figure b). His serum levels of C-reactive protein (CRP), lactate dehydrogenase (LDH), Krebs von den Lungen-6 (KL-6), and surfactant protein-D (SP-D) were elevated (CRP 25.77 mg/dL, LDH 980 U/L, KL-6 1469 U/mL, and SP-D $936.2 \mathrm{ng} / \mathrm{mL}$ ) (Table 2). Assays were negative for serum $\beta$-D-glucan, serum interferon-gamma release, a loopmediated isothermal amplification for Mycoplasma pneumoniae, polymerase chain reaction for Bordetella pertussis, and serum pertussis toxin antibody. Streptococcus pneumoniae and Legionella pneumophila urinary antigen tests, sputum Gram stain, and sputum acid-fast bacillus tests were also negative. His blood and sputum produced negative bacterial cultures.

Endotracheal aspiration and bronchoalveolar lavage could not be performed because he showed severe hypoxemia and respiratory failure requiring mechanical ventilation, but he refused invasive ventilation. He was not taking any dietary supplements and had not undergone radiation therapy. He did not have lower limb edema or jugular venous distention on a physical examination, and chest $\mathrm{CT}$ did not show pleural effusion on either side. An electrocardiogram and transthoracic echocardiography findings did not indicate cardiac failure, tamponade, arrhythmia, or ischemic disease. In addition, he had no bloody sputum, hemoptysis, or progression of anemia, and we noted no findings suggesting alveolar bleeding.

We diagnosed him with AE-IIP using the definition of AE-IPF (2) because there is no clear definition of AE-IIP and previous reports on AE-IIP used the definition of AEIPF (5). He was administered corticosteroid pulse therapy and antibiotics. He underwent noninvasive positive-pressure ventilation. After successful treatment, he was discharged with oxygen therapy at a flow rate of $4 \mathrm{~L} / \mathrm{min}$.

\section{Discussion}

This case demonstrated that cardiac ablation can cause AE-IIP, which has a mortality rate of $60-85 \%$ (2). This initial report of cardiac ablation causing AE-IIP produced two main findings. First, a preoperative evaluation for the presence of IIP by chest CT is important because cardiac ablation can cause AE-IIP. This finding aligns with recommendations to perform chest CT when physicians suspect inter- 
Table 2. Laboratory Data before Catheter Ablation and on Admission.

\begin{tabular}{|c|c|c|c|}
\hline & & Before & At admission \\
\hline \multicolumn{4}{|l|}{ Hematology } \\
\hline WBC & $(/ \mu \mathrm{L})$ & 7,300 & 16,100 \\
\hline Neutrophils & $(\%)$ & 53.7 & 83.0 \\
\hline Lymphocytes & $(\%)$ & 31.0 & 8.5 \\
\hline Monocytes & $(\%)$ & 6.6 & 7.5 \\
\hline Eosinophils & $(\%)$ & 7.7 & 0 \\
\hline Basophils & $(\%)$ & 1.0 & 0.5 \\
\hline $\mathrm{RBC}$ & $\left(10^{4} / \mu \mathrm{L}\right)$ & 401 & 347 \\
\hline $\mathrm{Hb}$ & $(\mathrm{g} / \mathrm{dL})$ & 13.0 & 10.9 \\
\hline $\mathrm{Ht}$ & $(\%)$ & 38.4 & 33.1 \\
\hline Plt & $\left(10^{4} / \mu \mathrm{L}\right)$ & 33.6 & 36.2 \\
\hline \multicolumn{4}{|c|}{ Blood chemistry } \\
\hline AST & $(\mathrm{U} / \mathrm{L})$ & 22 & 79 \\
\hline ALT & $(\mathrm{U} / \mathrm{L})$ & 11 & 39 \\
\hline T-Bil & $(\mathrm{mg} / \mathrm{dL})$ & 0.9 & 2.0 \\
\hline LDH & (U/L) & 253 & 980 \\
\hline $\mathrm{Na}$ & $(\mathrm{mEq} / \mathrm{L})$ & 141.1 & 136.3 \\
\hline $\mathrm{K}$ & $(\mathrm{mEq} / \mathrm{L})$ & 4.6 & 4.3 \\
\hline $\mathrm{Cl}$ & $(\mathrm{mEq} / \mathrm{L})$ & 106.6 & 102.1 \\
\hline BUN & $(\mathrm{mg} / \mathrm{dL})$ & 22.0 & 41.0 \\
\hline $\mathrm{Cr}$ & $(\mathrm{mg} / \mathrm{dL})$ & 0.89 & 1.46 \\
\hline TP & $(\mathrm{g} / \mathrm{dL})$ & 7.3 & 7.3 \\
\hline Alb & $(\mathrm{g} / \mathrm{dL})$ & 3.6 & 3.4 \\
\hline CRP & $(\mathrm{mg} / \mathrm{dL})$ & 0.22 & 25.77 \\
\hline PCT & (ng/mL) & & 1.02 \\
\hline KL-6 & $(\mathrm{U} / \mathrm{mL})$ & 789 & 1,469 \\
\hline SP-D & $(\mathrm{ng} / \mathrm{mL})$ & 413.0 & 936.2 \\
\hline BNP & $(\mathrm{pg} / \mathrm{mL})$ & 135.6 & 553.2 \\
\hline
\end{tabular}

WBC: white blood cell, RBC: red blood cell, Hb: hemoglobin, Ht: hematocrit, Plt: platelet, AST: aspartate transaminase, ALT: alanine transaminase, T-Bil: total bilirubin, LDH: lactate dehydrogenase, Na: sodium, K: potassium, $\mathrm{Cl}$ : chloride, BUN: blood urea nitrogen, Cr: creatinine, TP: total protein, Alb: albumin, CRP: C-reactive protein, PCT: procalcitonin, KL-6: Krebs von den lungen-6, SP-D: surfactant protein-D, BNP: brain natriuretic peptide

stitial pneumonia based on preoperative symptoms and the respiratory function. This is important because inflammatory responses to surgical intervention, exposure to high oxygen concentrations, and overdistension of the lung during positive-pressure ventilation may trigger IIP progression $(2,6)$. Cardiac computed tomographic angiography (CCTA) is also recommended for pulmonary vein mapping to rule out left atrial appendage thrombus and to allow for the detailed, simultaneous evaluation of partial lung fields and associated structures (7). Our recommendation to evaluate lung field shadows corresponds to a previous study that recommended additional follow-up for patients with noncardiac abnormalities on CCTA (7).

Second, AE-IIP should be considered a possible cause of respiratory symptoms and failure after cardiac ablation. Complications associated with ablation have a reported incidence of $3-6 \%$. They include cardiac tamponade (1.3\%), thromboembolic events $(0.4 \%)$, atrial-esophageal fistula $(0.2 \%)$, and respiratory complications $(1.3 \%)$, such as pulmonary vein stenosis, drug-induced pneumonia, and respiratory infections (8-10). Although acute respiratory distress syndrome (ARDS) was reported as a cause of death associated with cardiac ablation, the mechanism, and frequency underlying ARDS are unclear (9). It is important to note that not only heart failure, ARDS, and respiratory infections but also AE-IIP can cause respiratory failure and new diffuse bilateral ground-glass opacities or consolidation on chest CT after ablation. This is because high-dose steroid therapy is a reasonable choice for patients with AE-IIP but is an uncommon treatment for heart failure, ARDS, and pulmonary infections (2). The prognosis of AE-IIP also differs from that of heart failure and infectious diseases due to the existence of AE-IIP-related ventilator withdrawal difficulties after endotracheal intubation (11).

The mechanisms underlying AE-fibrotic IIP remain unclear. Reports have shown that mediators, such as transforming growth factor (TGF $\beta$ ) and matrix metalloproteases (MMP) - both regulators of extracellular matrix turnover and remodeling - contribute to epithelial damage and inflammation (11). In the MMP family, MMP2 and MMP9 are expressed in fibrotic IIP tissues (12). Reports have also shown that increases in TGF $\beta$ activated by MMP2 and MMP9 may promote AE-IIP development (2). Elevated MMP2, MMP9, and TGF $\beta$ levels were also observed in the heart tissue and within the systemic circulation after cardiac ablation for $\mathrm{AF}$ (13). In the present case, MMP2, MMP9, and TGF $\beta$ activated by cardiac ablation may have contributed to AE-IIP.

Several limitations associated with the present study warrant mention. We were unable to completely rule out infections and alveolar bleeding because we did not perform endotracheal aspirate or bronchoalveolar lavage, and we were unable to completely rule out drug-induced lung injury.

\section{Conclusion}

Cardiac ablation, a widely used treatment for arrhythmia, can cause AE-IIP. The present findings underscored the importance of performing a clinical interview, physical examination, and chest radiography in patients with IIP before cardiac ablation. AE-IIP should also be considered a possible complication of cardiac ablation. Further investigations will be necessary to determine the incidence and severity of AE-IIP after cardiac ablation.

The authors state that they have no Conflict of Interest (COI).

\section{References}

1. Raghu G, Collard HR, Egan JJ, et al. An official ATS/ERS/JRS/ ALAT statement: idiopathic pulmonary fibrosis: evidence-based guidelines for diagnosis and management. Am J Respir Crit Care Med 186: 788-824, 2011.

2. Collard HR, Moore BB, Flaherty KR, et al. Acute exacerbation of idiopathic pulmonary fibrosis. Am J Respir Crit Care Med 176: 
636-643, 2007.

3. Ryerson CJ, Cottin V, Brown KK, Collard HR. Acute exacerbation of idiopathic pulmonary fibrosis: shifting the paradigm. Eur Respir 46: 512-520, 2015.

4. Kuck KH, Brugada J, Fürnkranz A, et al. Cryoballoon or radiofrequency ablation for paroxysmal atrial fibrillation. N Engl J Med 374: 2235-2245, 2016.

5. Gabriela L, Jürgen B. Acute exacerbation in interstitial lung disease. Front Med 176: 4, 2017.

6. Kubota T, Miyata A. Postoperative respiratory failure caused by acute exacerbation of idiopathic interstitial pneumonia. J Anesth 25: 422-425, 2011

7. Budoff MJ, Fischer H, Gopal A. Incidental findings with cardiac CT evaluation: should we read beyond the heart? Catheter Cardiovasc Interv 68: 965-973, 2006.

8. Deshmukh A, Patel NJ, Pant SP, et al. In-hospital complications associated with catheter ablation of atrial fibrillation in the United States between 2000 and 2010: analysis of 93801 procedures. Circulation 128: 2104-2112, 2013.

9. Dagres N, Hindricks G, Kottkamp H, et al. Complications of atrial fibrillation ablation in a high-volume center in 1,000 procedures: still cause for concern? J Cardiovasc Electrophysiol 20: 10141019, 2009

10. Riccardo C, Hugh C, Shih AC, et al. Prevalence and causes of fatal outcome in catheter ablation of atrial fibrillation. J Am Coll Cardiol 12: 1798-1803, 2009.

11. Suga M, Iyonaga K, Okamoto T, et al. Characteristic elevation of matrix metalloproteinase activity in idiopathic interstitial pneumonias. Am J Respir Crit Care Med 162: 1949-1956, 2000.

12. Selman M, Ruiz V, Cabrera S, et al. TIMP-1, $-2,-3$, and -4 in idiopathic pulmonary fibrosis. A prevailing nondegradative lung microenvironment? Am J Physiol Lung Cell Mol Physiol 279: 562574, 2000.

13. Okumura $Y$, Watanabe I, Nakai $T$, et al. Impact of biomarkers of inflammation and extracellular matrix turnover on the outcome of atrial fibrillation ablation: importance of matrix metalloproteinase2 as a predictor of atrial fibrillation recurrence. J Cardiovasc Electrophysiol 22: 987-993, 2011.

The Internal Medicine is an Open Access journal distributed under the Creative Commons Attribution-NonCommercial-NoDerivatives 4.0 International License. To view the details of this license, please visit (https://creativecommons.org/licenses/ by-nc-nd/4.0/).

(C) 2021 The Japanese Society of Internal Medicine

Intern Med 60: 3589-3592, 2021 